\title{
Comportamento fenológico de espécies lenhosas em um cerrado sentido restrito de Brasília, DF
}

\author{
EDDIE LENZA ${ }^{1,2}$ e CARLOS AUGUSTO KLINK ${ }^{1}$
}

(recebido: 24 de março de 2005; aceito: 9 de novembro de 2006)

\begin{abstract}
Phenological behavior of woody species in a "cerrado" sensu stricto of Brasília, DF). In this study we followed the phenology of 19 woody species of a cerrado sensu stricto in the IBGE Ecological Reserve (15 $55^{\prime} 06^{\prime \prime}-15^{\circ} 57^{\prime} 57^{\prime \prime} \mathrm{S}$ and 4751'22"-4754'07' W), in Brasília, Federal District, Brazil. Phenological observations were made every fortnight between August 2000 and October 2003. The vegetation studied is a seasonal semi-deciduous wood savanna, where a hot wet season (from October through to April) is followed by a cool dry season (from May through to September). Vegetation canopy is reduced during the dry season and foliage cover reaches a minimum of $50 \%$ at the end of the dry season. Even though new leaves and flowers appear along the year, their production is intensified during the transition between the dry and wet seasons. According to the vegetative phenology, four groups were identified: four evergreen species with continuous growth, five evergreen species with seasonal growth, eight brevideciduous species and two deciduous species. The evergreen species with continuous growth produced leaves along the wet season, while the remaining groups produced leaves more intensely at the end of the dry season. Fruit maturation of autochoric and anemochoric species occurred within the dry season; zoochoric species dispersed seeds mainly during the wet season. Soil water availability seemed not to have restricted leaf production and reproduction of most species since the peak of flushing and blooming was at the end of the dry season. However, endogenous factors such as leaf longevity and internal water balance, as well as exogenous factors such as evaporative demand and irradiation seem to influence the phenological patterns observed in this vegetation.
\end{abstract}

Key words - "cerrado", climatic seasonality, neotropical savanna, phenology, woody species

RESUMO - (Comportamento fenológico de espécies lenhosas em um cerrado sentido restrito de Brasília, DF). Neste estudo nós acompanhamos a fenologia de 19 espécies lenhosas de um cerrado sentido restrito na Reserva Ecológica do IBGE

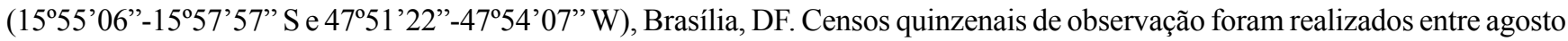
de 2000 e outubro de 2003. A região apresenta uma evidente sazonalidade climática, com um período quente e chuvoso entre outubro e abril e um período frio e seco entre maio e setembro. A vegetação estudada foi classificada como sazonal semidecidual. A cobertura de copa da vegetação apresentou redução a partir do início do período seco, alcançando os menores valores de folhagem (cerca de 50\%) no final deste período. Foram observadas espécies brotando e florescendo ao longo de todo ano, entretanto a produção de novas folhas e a floração foi intensa na transição entre o período seco e o chuvoso. Quatro grupos fenológicos vegetativos foram identificados: quatro espécies sempre verdes com crescimento contínuo, cinco sempre verdes com crescimento sazonal, oito brevidecíduas e duas decíduas. As espécies sempre verdes com crescimento contínuo produziram folhas ao longo do período chuvoso, enquanto as espécies dos demais grupos fenológicos formaram folhas intensamente no final do período seco. A maturação de frutos das espécies com dispersão autocórica e anemocórica ocorreu no período seco e aquelas com dispersão zoocórica dispersaram as sementes principalmente durante o período chuvoso. A disponibilidade de água no solo parece não ter restringido a produção de folhas e a reprodução na maioria das espécies, uma vez que os picos de brotação e de floração foram concentrados no fim do período seco. No entanto, fatores endógenos, como longevidade foliar e balanço hídrico interno; e exógenos, como demanda evaporativa e irradiação parecem estar relacionados aos padrões fenológicos observados.

Palavras-chave - cerrado, espécies lenhosas, fenologia, savana neotropical, sazonalidade climática

\section{Introdução}

Uma das características marcantes do cerrado é a sazonalidade na temperatura e precipitação ao longo do

\footnotetext{
1. Universidade de Brasília, Instituto de Ciências Biológicas, Departamento de Ecologia, Caixa Postal 04621, 70910-900 Brasília, DF, Brasil.

2. Autor para Correspondência: eddie@unb.br
}

ano, que define a existência de dois períodos climáticos bem marcados: um quente e úmido e outro frio e seco (Eiten 1972, Sarmiento 1984). No período seco ocorrem incêndios freqüentes (Miranda et al. 2002) e o solo está sujeito a um déficit hídrico sazonal nas camadas mais superficiais (Franco 2002).

A combinação entre as flutuações climáticas sazonais, as características físico-químicas dos solos e a ocorrência de queimadas determinam a distribuição, a estrutura e o funcionamento das diferentes formações 
vegetais deste bioma (Eiten 1972, Furley \& Ratter 1988). As formações savânicas predominam ao longo de toda extensão do cerrado e essas, conjuntamente chamadas de cerrado sensu lato por Oliveira Filho \& Ratter (2002), são caracterizadas por dois estratos distintos. O primeiro é representado por uma vegetação herbáceograminosa e o segundo por espécies arbóreas e arbustivas, cujas densidades e altura são determinadas pela fertilidade e profundidade do solo, altura do lençol freático e freqüência do fogo (Eiten 1972, Furley \& Ratter 1988, Moreira 2000).

O ciclo de vida das espécies arbóreo-arbustivas do cerrado é perene e as espécies possuem mecanismos que facilitam sua sobrevivência e reprodução durante o período seco, quando aumenta a restrição hídrica (Sarmiento 1984, Oliveira 1998). A existência de estruturas que conservam água, como os xilopódios, ou de um sistema radicular bem desenvolvido, que muitas vezes pode alcançar camadas mais profundas do solo, onde há água disponível (Eiten 1972, Jackson et al. 1999, Oliveira et al. 2005), são responsáveis pela manutenção de estruturas aéreas de sustentação e fotossintetizantes ao longo do ano (Eiten 1972, Sarmiento 1984).

As espécies do estrato lenhoso possuem diferentes estratégias fenológicas que lhes permite superar o estresse hídrico sazonal (Oliveira 1998). As espécies sempre verdes se mantêm com folhas na copa o ano todo (Sarmiento et al. 1985, Oliveira 1998, Franco et al. 2005), enquanto que espécies brevidecíduas e decíduas perdem completamente a folhagem por certo tempo durante o período seco (Sarmiento et al. 1985, Miranda 1995, Morais et al. 1995, Franco et al. 2005). A renovação da folhagem nos dois grupos está normalmente associada ao período seco (Sarmiento et al. 1985, Miranda 1995, Oliveira \& Gibbs 2000). A floração destas espécies também se concentra no período seco ou na transição entre o período seco e o chuvoso (Oliveira \& Gibbs 2000, Batalha \& Mantovani 2000). Estes são indícios de que tanto a produção de folhas quanto a reprodução de espécies lenhosas do cerrado não são limitadas pela redução de água no solo durante o período seco (Batalha \& Mantovani 2000, Franco et al. 2005).

Estudos fenológicos desenvolvidos em outras comunidades de cerrado demonstram que é possível predizer que os eventos fenológicos vegetativos e reprodutivos das espécies lenhosas determinam a natureza sazonal da comunidade lenhosas do cerrado apesar das espécies apresentarem distintos comportamentos fenológicos (Mantovani \& Martins 1988, Miranda 1995, Jackson et al. 1999, Batalha \&
Mantovani 2000, Oliveira \& Gibbs 2000). Neste sentido, o presente estudo teve por objetivos: 1) caracterizar o comportamento fenológico vegetativo e reprodutivo de 19 espécies lenhosas representativas de um cerrado sentido restrito localizado na Reserva Ecológica do IBGE, Brasília, Distrito Federal; e 2) identificar grupos fenológicos vegetativos para as espécies, levando em consideração aspectos temporais das distintas fenofases, conforme proposto na literatura para espécies de ambientes savânicos (Sarmiento \& Monasterio 1983, Franco et al. 2005).

\section{Material e métodos}

Este estudo foi conduzido em uma área de cerrado sentido restrito (Ribeiro \& Walter 1998, Oliveira Filho \& Ratter 2002) localizado na Reserva Ecológica do IBGE (Recor), situada a cerca de $35 \mathrm{~km}$ ao sul do centro de Brasília, DF

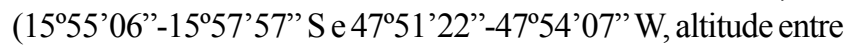
$1.045 \mathrm{e} 1.146 \mathrm{~m})$.

A precipitação média anual na Recor foi de $1.438 \mathrm{~mm}$ no período entre 1992 e 2002, com dois períodos distintos, o primeiro chuvoso e quente, entre outubro e abril, e o segundo seco e um pouco mais frio, entre maio e setembro. Durante o período de estudo foi observada marcada sazonalidade climática, abrangendo três períodos secos e três períodos chuvosos completos (Fonte: Estação Meteorológica da Recor - www.recor.org.br). A precipitação total variou entre os três períodos chuvosos, sendo de $1.478 \mathrm{~mm}$ para o período chuvoso de 2000/2001 (1.254 mm entre outubro e abril); $1.219 \mathrm{~mm}$ para 2001/2002 (1.142 mm entre outubro e abril); e $1.228 \mathrm{~mm}$ para $2002 / 2003$ (1.031 mm entre outubro e abril). As temperaturas mínimas mensais variaram entre $15^{\circ} \mathrm{Ce} 18^{\circ} \mathrm{C}$ no período chuvoso (outubro a abril) e entre $11^{\circ} \mathrm{C}$ e $12^{\circ} \mathrm{C} \mathrm{em}$ meados do período seco (junho e julho). Ao longo do ano as temperaturas médias mensais variaram entre $20^{\circ} \mathrm{Ce} 25^{\circ} \mathrm{C}$ e as máximas mensais entre $26^{\circ} \mathrm{C}$ e $30^{\circ} \mathrm{C}$. A média mensal da umidade relativa do ar se manteve acima de $70 \%$ durante praticamente todo o período chuvoso e entre $50 \%$ e $60 \%$ durante o período seco. A evaporação mensal foi intensa durante o período seco e início do período chuvoso com valores mais elevados observados geralmente entre os meses de julho e outubro (entre $150 \mathrm{~mm}$ e $210 \mathrm{~mm}$ ) (figura 1). Os solos são predominantemente Latossolos vermelho-escuro e vermelho-amarelo, profundos, bem drenados, com alto teor de argila, baixa fertilidade, alta toxidez de alumínio (Haridasan 1993) e com topografia suave.

Ao todo foi estudada a fenologia de 226 indivíduos reprodutivos com circunferência do tronco $\geq 10 \mathrm{~cm}$ no nível do solo, pertencentes a 19 espécies e 14 famílias botânicas: Acosmium dasycarpum (Vog.) Yakovl., Blepharocalyx salicifolius (Kunth) O. Berg., Byrsonima verbascifolia (L.) Rich. ex. A.L. Juss., Casearia sylvestris Sw., Connarus suberosus Planch., Dalbergia miscolobium Benth., Davilla 
elliptica A. St.-Hil., Dimorphandra mollis Benth., Erythroxylum suberosum A. St.-Hil., Kielmeyera coriacea (Spreng.) Mart., Miconia albicans (Sw.) Triana, Miconia fallax DC., Ouratea hexasperma (A. St.-Hil.) Baill., Qualea grandiflora Mart., Myrsine guianensis (Aubl.) Kuntze, Roupala montana Aubl., Rourea induta Planch., Stryphnodendron adstringens (Mart.) Cov. e Styrax ferrugineus Nees \& Mart. (tabela 1). Estas espécies apresentam geralmente ampla distribuição e elevada abundância tanto na área de domínio do bioma cerrado (Ratter et al. 2003) quanto em fisionomias de cerrado do Distrito Federal (Nunes et al. 2002) e da Recor (Andrade et al. 2002).

As observações fenológicas foram realizadas em intervalos quinzenais no período de agosto de 2000 a outubro
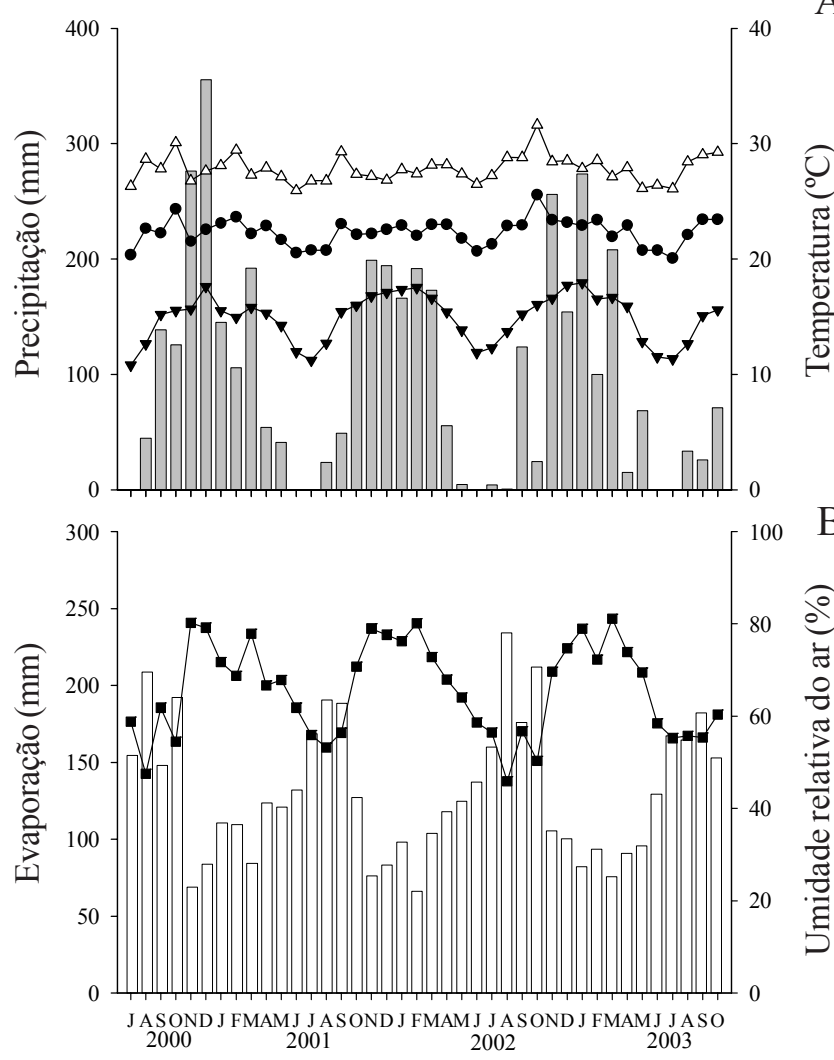

Figura 1. Clima da Reserva Ecológica do IBGE (Recor), Brasília, DF durante o período de estudo (agosto de 2000 a outubro de 2003). A. Precipitação média mensal ( $\square$ ) e valores máximo $(\triangle)$, médio $(\bullet)$ e mínimo $(\nabla)$ da temperatura mensal. B. Média mensal da evaporação ( $\square$ ) e da umidade relativa do ar (घ). Fonte: Estação Meteorológica da Recor (www.recor.org.br).

Figure 1. Climate at Reserva Ecológica do IBGE (Recor), Brasília, DF, during the study period (August 2000 through October 2003). A. Average monthly precipitation ( $\square$ ), maximum $(\triangle)$, average $(\bullet)$, and minimum $(\boldsymbol{\nabla})$ monthly temperature. B. Average monthly evaporation $(\square)$ and average relative humidity (匹). Data source: Recor meteorological station (www.recor.org.br). de 2003. Apenas em Dimorphadra mollis as observações foram realizadas até agosto de 2002, pois fortes ventos naquele mês derrubaram duas plantas, reduzindo para três indivíduos na população, número que consideramos pequeno para estudos fenológicos. Para cada espécie foi estimada a cobertura individual da copa, registrada a ocorrência de brotamento ou formação de folhas novas, presença de botões florais, flores e frutos maduros. A cobertura individual da copa foi estimada visualmente, em alguns casos com auxílio de binóculo, de acordo com as seguintes classes de cobertura de folhas (Fournier 1974): 0 - copa sem folhas; 1 - copa com cobertura de folhas entre $1 \%$ e $25 \%$; 2 - copa com cobertura entre $26 \%$ e $50 \%$; 3 - copa com cobertura entre $51 \%$ e $75 \%$; 4 - copa com cobertura entre $76 \%$ e $100 \%$. Usando as estimativas de cobertura individual da copa foi calculado o percentual de intensidade de cobertura de copa (PICP) (Fournier 1974) para as 19 espécies em conjunto e separadamente.

As espécies foram classificadas em grupos fenológicos vegetativos (Sarmiento \& Monasterio 1983, Franco et al. 2005) que se baseiam em aspectos temporais de ocorrência das fenofases de queda e produção de folhas, bem como no grau de deciduidade ou caducifolia dos indivíduos. A freqüência e a regularidade de ocorrência de floração das espécies foram caracterizadas de acordo com a classificação de Newstrom \& Frankie (1994). As síndromes de dispersão das espécies foram determinadas seguindo a classificação adotada por van der Pijl (1982), segundo a qual as espécies podem ser classificadas em três grandes grupos de acordo com as características das unidades de dispersão das sementes: 1) anemocoria dispersão pelo vento; 2) autocoria - auto-dispersão ou dispersão sem a ação de um agente dispersor; e 3) zoocoria dispersão por animais.

\section{Resultados}

Fenologia vegetativa - Quando analisadas conjuntamente, a produção e queda de folhas das 19 espécies estudadas foram marcadamente sazonais (figura 2). O percentual de intensidade de cobertura de copa (PICP) se manteve alto (próximo de 100\%) e constante ao longo de todo o período chuvoso até o início do período seco. O PICP decresceu a partir de junho e atingiu valores mínimos (entre $50 \%$ e $60 \%$ ) ao final do período seco, entre os meses de agosto e setembro.

A brotação foi observada ao longo de todo ano. Contudo, tanto a proporção de espécies quanto a de indivíduos em brotação foi menor durante a maior parte do período seco (entre maio e agosto) e os picos de brotação ocorreram em setembro e outubro. Conseqüentemente, a reconstituição da copa se deu na transição entre o período seco e o chuvoso (figura 2). 
Tabela 1. Espécies lenhosas acompanhadas nos estudos fenológicos de um cerrado sentido restrito na Reserva Ecológica do IBGE, $\mathrm{DF}$. (Hb. = hábito; $\mathrm{av}=$ árvore; $\mathrm{ab}=$ arbusto; $\mathrm{N}=$ número de indivíduos; $\mathrm{SF}=$ Média \pm desvio padrão em dias que os indivíduos permaneceram sem folhas durante o período seco; Disp. = Síndrome de dispersão; zoo = zoocórica; ane = anenocórica; aut $=$ autocórica).

Table 1. Woody species surveyed in phenological studies of a cerrado stricto sensu in the Reserva Ecológica do IBGE (Recor), Brasília, DF. (Hb. = growth habit; $\mathrm{av}=$ tree; $\mathrm{ab}=\operatorname{shrub;~} \mathrm{N}=$ number of individuals; $\mathrm{SF}=\mathrm{Mean} \pm$ standard deviation on the days when individuals had no leaves during the dry season; Disp. $=$ Dispersal syndrome; zoo $=$ zoochorous; ane $=$ anemochorous; aut $=$ autochorous).

\begin{tabular}{|c|c|c|c|c|c|c|c|}
\hline \multirow[t]{2}{*}{ Grupo fenológico vegetativo/espécie } & \multirow[t]{2}{*}{ Família } & \multirow[t]{2}{*}{$\mathrm{Hb}$. } & \multirow[t]{2}{*}{$\mathrm{N}$} & \multicolumn{3}{|c|}{$\mathrm{SF}$} & \multirow[t]{2}{*}{ Disp } \\
\hline & & & & 2001 & 2002 & 2003 & \\
\hline \multicolumn{8}{|l|}{ Sempre verde com crescimento contínuo (SVC) } \\
\hline Miconia albicans (Sw.) Triana & Melastomataceae & $a b$ & 11 & 0 & 0 & 0 & zoo \\
\hline M. fallax DC. & Melastomataceae & $a b$ & 06 & 0 & 0 & 0 & zoo \\
\hline Connarus suberosus Planch. & Connaraceae & $a b$ & 07 & 0 & 0 & 0 & zoo \\
\hline Myrsine guianensis (Aubl.) Kuntze & Myrsinaceae & av & 16 & 0 & 0 & 0 & zoo \\
\hline \multicolumn{8}{|l|}{ Sempre verde com crescimento sazonal (SVS) } \\
\hline Roupala montana Aubl. & Proteaceae & av & 18 & 0 & 0 & 0 & ane \\
\hline Ouratea hexasperma (A. St.-Hil.) Baill. & Ochnaceae & $a b$ & 14 & 0 & 0 & 0 & zoo \\
\hline Styrax ferrugineus Nees \& Mart. & Styracaceae & av & 16 & 0 & 0 & 0 & zoo \\
\hline Rourea induta Planch. & Connaracea & $a b$ & 18 & 0 & 0 & 0 & zoo \\
\hline Casearia sylvestris $\mathrm{Sw}$. & Flacourtiacae & $a b$ & 11 & 0 & $3 \pm 9$ & 0 & zoo \\
\hline \multicolumn{8}{|l|}{ Brevidecíduo (BDC) } \\
\hline Blepharocalyx salicifolius (Kunth) O.Berg. & Myrtaceae & av & 12 & 0 & 0 & 0 & zoo \\
\hline Byrsonima verbascifolia (L.) Rich. ex. A. L. Juss. & Malpighiaceae & av & 13 & $1 \pm 5$ & $2 \pm 5$ & 0 & zoo \\
\hline Stryphnodendron adstringens (Mart.) Cov. & Leguminosae & av & 11 & 0 & $1 \pm 5$ & $4 \pm 10$ & aut \\
\hline Davilla elliptica A. St.-Hil. & Dilleniaceae & $\mathrm{ab}$ & 20 & $1 \pm 6$ & 0 & $2 \pm 10$ & zoo \\
\hline Erythroxylum suberosum A. St.-Hil. & Erythroxylaceae & $a b$ & 08 & $4 \pm 11$ & $2 \pm 5$ & $2 \pm 5$ & zoo \\
\hline Dimorphandra mollis Benth. & Leguminosae & av & 05 & $7 \pm 10$ & $10 \pm 12$ & - & aut \\
\hline Qualea grandiflora Mart. & Vochysiaceae & av & 06 & $6 \pm 9$ & $7 \pm 8$ & $7 \pm 8$ & ane \\
\hline Dalbergia miscolobium Benth. & Leguminosae & av & 18 & $13 \pm 10$ & $9 \pm 10$ & $9 \pm 8$ & aut \\
\hline \multicolumn{8}{|l|}{ Decíduo (DEC) } \\
\hline Acosmium dasycarpum (Vog.) Yakovl. & Leguminosae & $a b$ & 08 & $23 \pm 11$ & $27 \pm 23$ & $16 \pm 13$ & aut \\
\hline Kielmeyera coriacea (Spreng.) Mart. & Clusiaceae & av & 08 & $25 \pm 13$ & $20 \pm 11$ & $26 \pm 17$ & ane \\
\hline
\end{tabular}

Dentre as espécies estudadas, quatro grupos fenológicos vegetativos foram identificados: quatro espécies sempre verdes com crescimento contínuo, cinco sempre verdes com crescimento sazonal, oito brevidecíduas e duas decíduas (tabela 1). As principais características de cada grupo fenológico são apresentadas a seguir.

Espécies sempre verdes com crescimento contínuo (SVC) - As espécies deste grupo caracterizaram-se por uma ligeira redução no PICP ao longo do ano (geralmente acima de $80 \%$ ), produção de folhas novas por períodos prolongados (figura 3) e por um período pouco definido de substituição da folhagem.

A brotação de Miconia albicans foi interrompida apenas por um curto período no final da seca, entre agosto e setembro, enquanto não houve formação de novas folhas durante quase todo o período seco, entre junho e setembro, na espécie Miconia fallax (figura 3). Não houve um período definido de renovação da copa para estas duas espécies, ou seja, elas foram tipicamente SVC.

A brotação em Connarus suberosus foi reduzida durante o período chuvoso, principalmente entre os meses de dezembro e março. Entre os meses de agosto e outubro foi observada intensa atividade de perda e produção de folhas, entretanto houve pequena redução do PICP (figura 3) e nem todas as folhas foram substituídas neste período. Por esta razão a espécie também foi classificada como SVC.

A fenologia de Myrsine guianensis foi marcada por dois eventos anuais de perda e produção de folhas. O primeiro ocorreu na transição entre os períodos seco e chuvoso, quando a redução da copa foi mais acentuada 


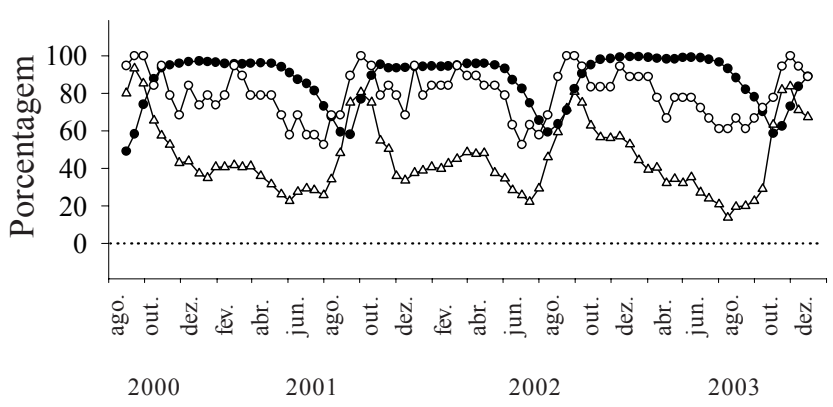

Figura 2. Aspectos fenológicos vegetativos de 19 espécies lenhosas de um cerrado sentido restrito na Reserva Ecológica do IBGE (Recor), Brasília, DF. Porcentagem de cobertura de copa $(\bullet)$; porcentagem de espécies brotando (O); porcentagem de indivíduos brotando $(\triangle)$.

Figure 2. Vegetative phenology of 19 woody species of a "cerrado" stricto sensu of Reserva Ecológica do IBGE (Recor), Brasília, DF. Percentage of canopy cover $(\bullet)$; percentage of species producing buds $(\mathrm{O})$; percentage of plants producing buds $(\triangle)$.

(PICP entre $30 \%$ e $40 \%$ ) e seguida de troca de quase toda folhagem no início do período chuvoso, entre outubro e dezembro. O segundo evento ocorreu dentro do período chuvoso com redução da copa menos evidente (PICP entre $80 \%$ e $90 \%$ ) e substituição apenas parcial da folhagem (figura 3). Embora tenha havido acentuada redução na cobertura de copa durante o primeiro evento, M. guianensis foi considerada como SVC pois sua brotação ocorreu ao longo do período chuvoso.

Espécies sempre verdes com crescimento sazonal (SVS) - Uma característica marcante das espécies SVC é a produção de uma nova coorte de folhas que antecede, ou ocorre concomitantemente, a perda da coorte de folhas velhas. Assim, as plantas continham folhas adultas mesmo durante o período de substituição da copa. Este grupo foi representado pelas espécies Roupala montana, Ouratea hexasperma, Styrax ferrugineus, Rourea induta e Casearia sylvestris, cuja produção e queda de folhas foram marcadamente sazonais (figura 4). Houve redução na cobertura da copa na segunda metade do período seco (PICP entre $50 \%$ e $70 \%$ ). A reconstituição da copa ocorreu na transição entre os períodos seco e chuvoso, quando quase todos os indivíduos destas cinco espécies estavam brotando intensamente (figura 4). Nos demais meses do ano a produção de folhas foi reduzida e geralmente restrita a alguns indivíduos (figura 4) que produziram um número pequeno de folhas jovens (Lenza 2005).

No transcorrer deste estudo os indivíduos de Roupala montana sofreram ataques específicos e sincrônicos de lagartas de Lepidóptera da Família
Notondotidae. As folhas produzidas entre setembro e outubro de 2001 foram consumidas pelas lagartas no mês de novembro, o que provocou redução acentuada da copa em todos os indivíduos dessa espécie. Como conseqüência houve intensa produção de folhas entre janeiro e julho de 2002 e recomposição da copa perdida (figura 4).

Espécies brevidecíduas (BDC) - A característica mais marcante das espécies deste grupo é a substituição completa da copa durante o período seco e um período curto, no qual os indivíduos permaneceram sem folhas ou possuíam apenas poucas folhas em formação ou senescentes. Durante o mês de setembro e início de

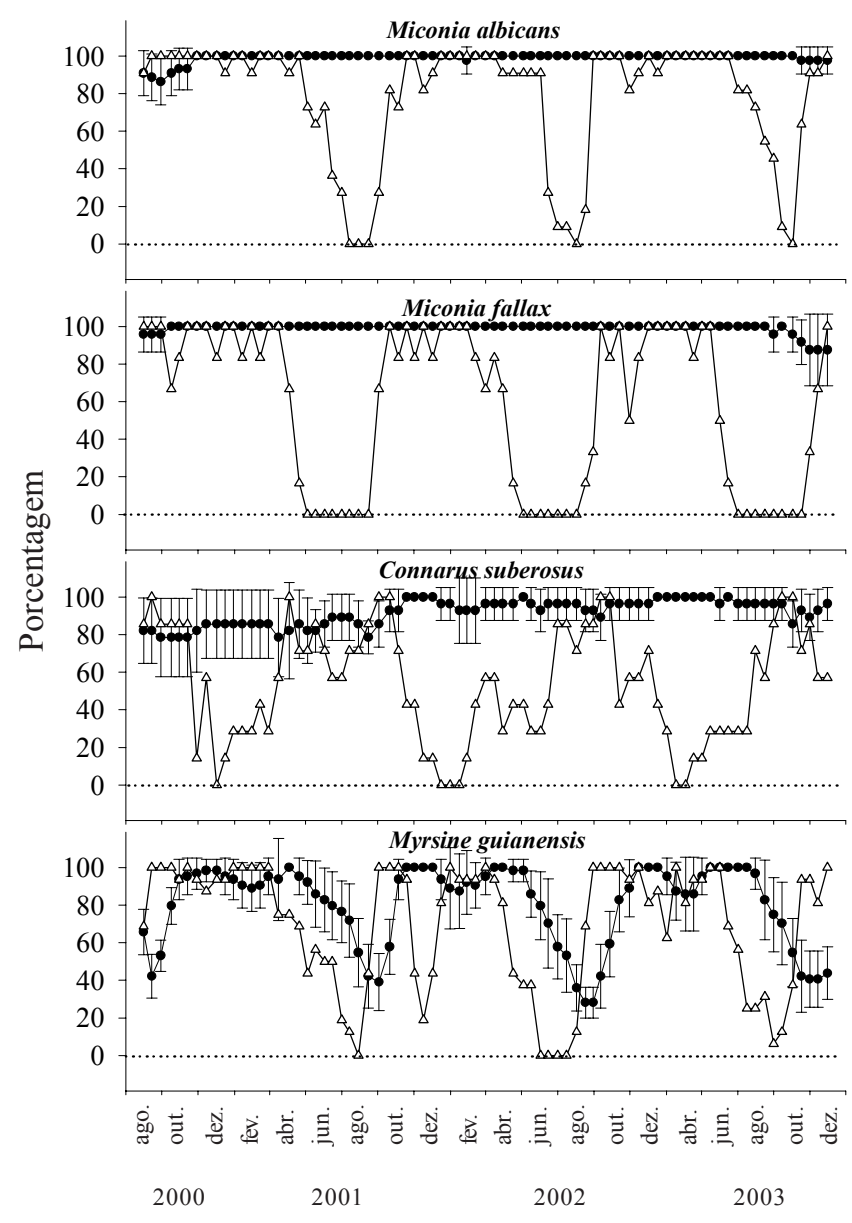

Figura 3. Porcentagem de cobertura de copa \pm desvio padrão $(\bullet)$ e de indivíduos brotando $(\triangle)$ de espécies lenhosas sempre verdes com crescimento contínuo (SVC) de um cerrado sentido restrito na Reserva Ecológica do IBGE (Recor), Brasília, DF.

Figure 3. Percentage of canopy cover \pm standard deviation $(\bullet)$ and percentage of plants producing buds $(\triangle)$ of evergreen woody species with continuous growth (SVC) of a "cerrado" stricto sensu of Reserva Ecológica do IBGE (Recor), Brasília, DF. 
outubro foi observada intensa brotação, culminando com a recomposição da copa no início do período chuvoso (figura 5). Alguns indivíduos destas espécies estavam em brotação fora do período de recomposição da copa (figura 5), entretanto foi observado no campo que estes produziam geralmente um número reduzido de folhas.

A produção de folhas novas ocorreu quando quase todas as folhas velhas já haviam sido perdidas nas

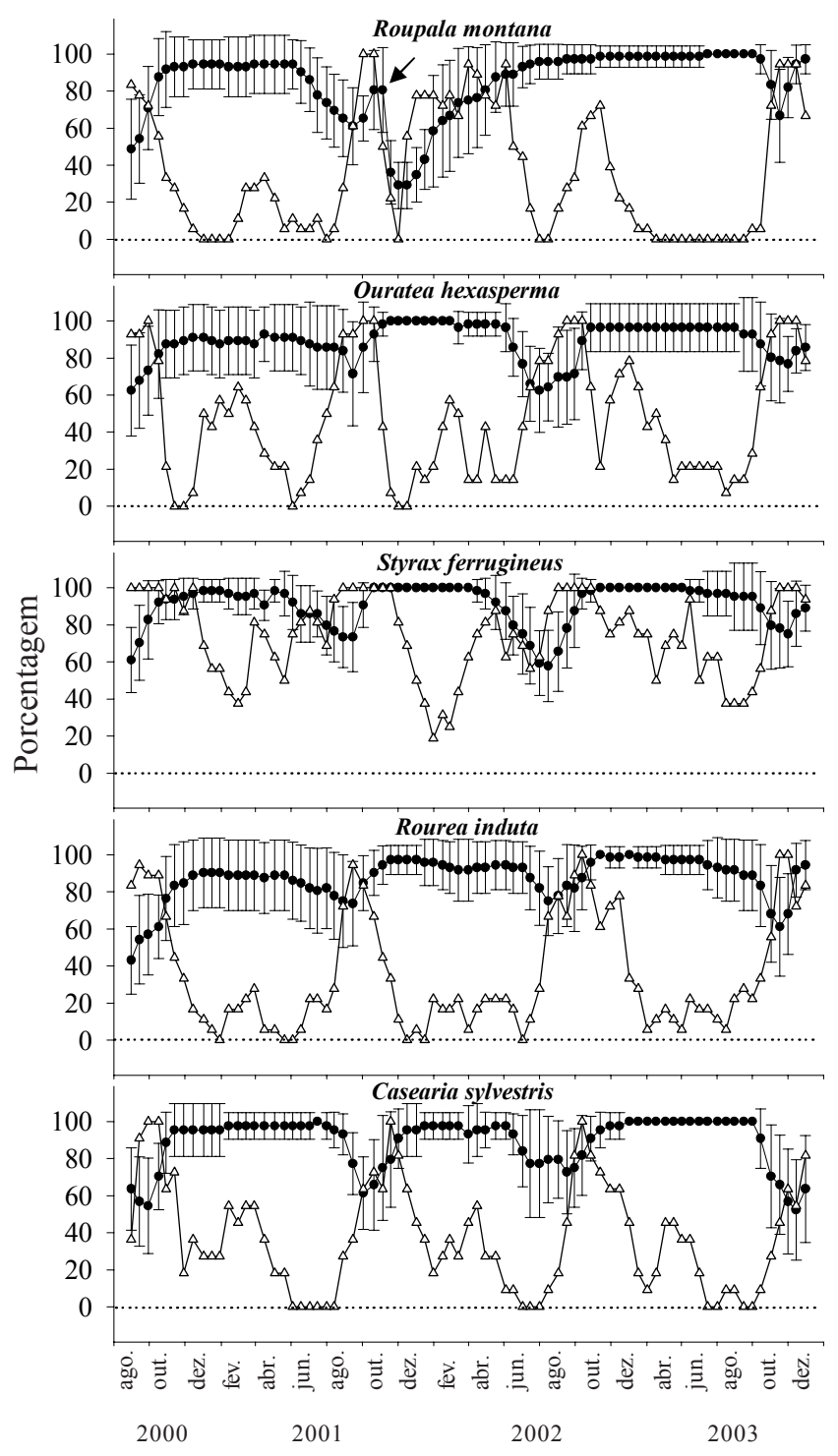

Figura 4. Porcentagem de cobertura de copa \pm desvio padrão $(\bullet)$ e de indivíduos brotando $(\triangle)$ de espécies lenhosas sempre verdes com crescimento sazonal (SVS) de um cerrado sentido restrito na Reserva Ecológica do IBGE (Recor), Brasília, DF. A seta indica período de moderada a intensa herbivoria.

Figure 4. Percentage of canopy cover \pm standard deviation $(\bullet)$ and percentage of plants producing buds $(\triangle)$ of evergreen woody species with seasonal growth (SVS) of a "cerrado" stricto sensu of Reserva Ecológica do IBGE (Recor), Brasília, DF. Arrow indicates moderated to strong herbivory. espécies Blepharocalyx salicifolius, Byrsonima verbascifolia, Stryphnodendron adstringens, Davilla elliptica e Erythroxylum suberosum, resultando em redução evidente no PICP das espécies (entre 30\% e $50 \%$ ) (figura 5). Ocasionalmente formam notadas variações no grau de deciduidade entre indivíduos de uma mesma espécie, como Byrsonima verbascifolia, Stryphnodendron adstringens e Davilla elliptica, cujas populações possuíam indivíduos com e sem deciduidade completa (tabela 1). Provavelmente as observações quinzenais adotadas neste estudo não foram suficientes para detectar períodos de deciduidade menores do que 15 dias em alguns indivíduos destas espécies.

Nas espécies Dimorphandra mollis, Qualea grandiflora e Dalbergia miscolobium os indivíduos permaneceram sem folhas durante um período médio, entre uma e duas semanas (tabela 1). Durante este período o PICP se reduziu para valores entre $10 \%$ e $30 \%$.

Folhas de Davilla elliptica sofreram ataque de lagartas de Pictotena sp. (Zygaenidae) em três eventos, nos quais houve redução da copa de pelo menos metade dos indivíduos acompanhados. O primeiro evento ocorreu em fevereiro e março de 2001, o segundo em janeiro e fevereiro de 2002 e o terceiro em julho de 2003. Nos anos de 2001 e 2002 as folhas adultas foram atacadas preferencialmente, o que resultou numa pequena, mas perceptível redução da copa. Em 2002 a produção de folhas novas e a recomposição da copa se deram logo após a herbivoria. No ano de 2003 a grande maioria das folhas atacadas estava em estágio avançado de senescência, ou seja, próximas à abscisão e, portanto, não ocorreu efeito perceptível da herbivoria na fenologia vegetativa (figura 5).

Espécies decíduas (DEC) - Tanto os indivíduos de Acosmium dasycarpum quanto de Kielmeyera coriacea, perderam completamente as folhas no final do período seco, de agosto a setembro. A produção de folhas foi sincrônica e intensa no final do período seco, no mês de setembro. O percentual de intensidade de cobertura de copa nas duas populações se reduziu drasticamente para valores entre $0 \%$ e $10 \%$ (figura 6). Os indivíduos permaneceram, em média, entre três e quatro semanas com deciduidade foliar completa. No entanto, os elevados desvios padrões observados em relação ao tempo médio de deciduidade foliar evidenciaram grandes variações no grau de deciduidade entre indivíduos de uma mesma população (tabela 1). Fenologia reprodutiva - A floração foi anual e regular para 17 das 19 espécies estudadas e apenas em Myrsine guianensis foi observada floração do tipo sub-anual, ou 
seja, ocorreu em diferentes momentos ao longo do ano (Newstrom \& Frankie 1994). Os indivíduos de Connarus suberosus não floresceram durante o período estudado (figura 7). Foram observadas espécies florescendo ao longo de todo ano, no entanto houve uma maior concentração da floração no período seco e na transição entre o período seco e o chuvoso (figura 7). Ocasionalmente, foram observados indivíduos isolados florescendo fora do período de floração da espécie. Nesses casos geralmente não foram gerados frutos (figura 7).

A maturação de frutos entre as espécies estudadas foi mais intensa entre os meses de agosto e janeiro, seguida de períodos de baixa maturação entre fevereiro e maio. Das 18 espécies que floresceram apenas Myrsine guianensis não produziu frutos (figura 7). Da

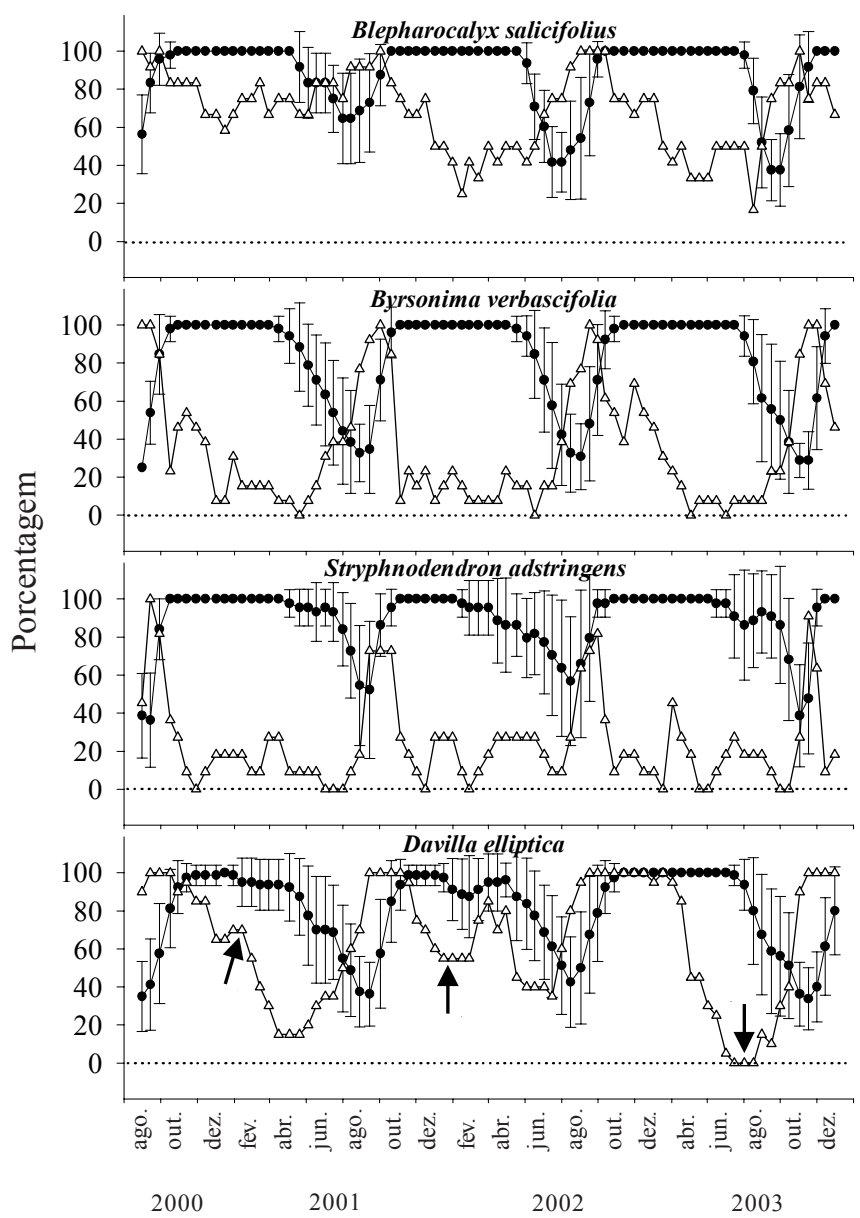

mesma forma que a floração, a maturação de frutos das espécies também seguiu um ciclo anual. As quatro espécies que dispersaram sementes no período seco apresentaram síndrome exclusivamente autocórica. Cinco espécies de síndrome zoocórica dispersaram sementes no período chuvoso. Das oito espécies que dispersaram sementes na transição entre o período seco e o chuvoso, três eram anemocóricas e cinco zoocóricas (tabela 1, figura 7 ).

\section{Discussão}

Comportamento fenológico das espécies - Quando se analisa o conjunto das 19 espécies estudadas, nota-se que a abscisão foliar ocorreu principalmente durante o período seco e foi sempre seguida de elevada brotação

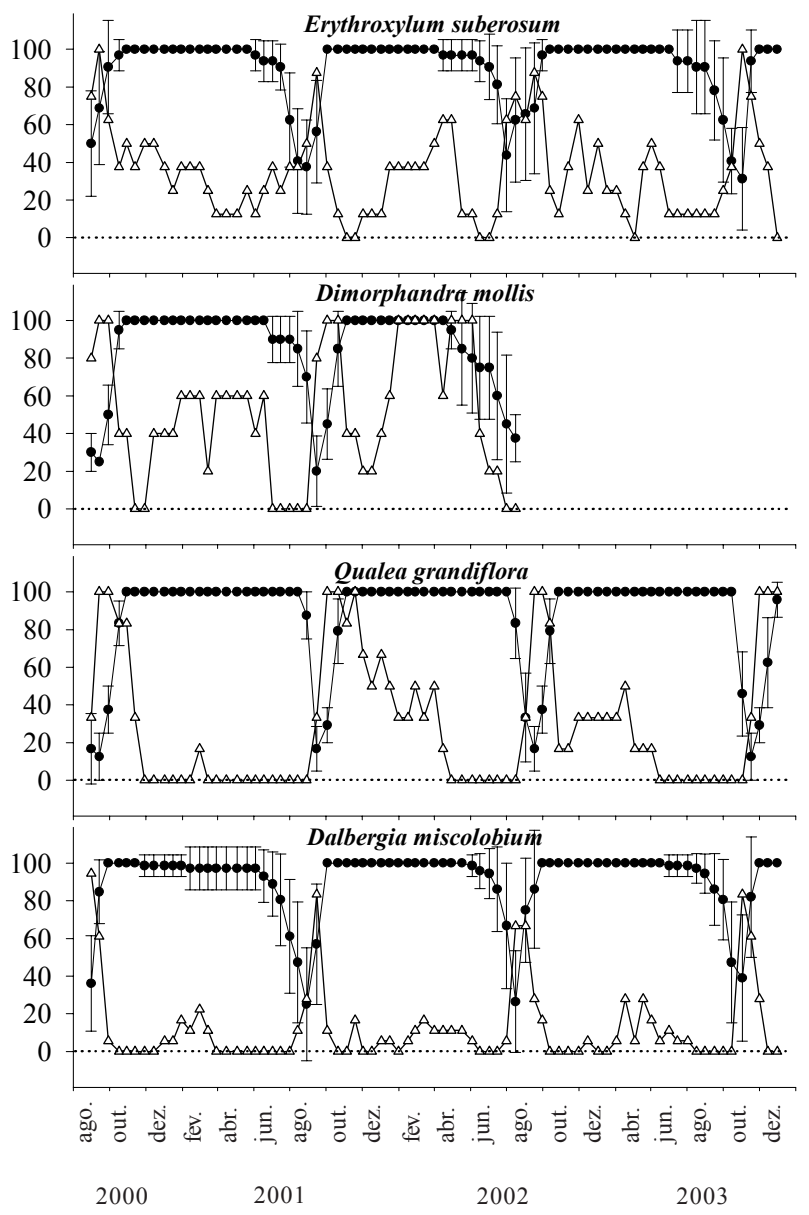

Figura 5. Porcentagem de cobertura de copa \pm desvio padrão $(\bullet)$ e de indivíduos brotando $(\triangle)$ de espécies lenhosas com comportamento fenológico brevidecíduo (BDC) de um cerrado sentido restrito na Reserva Ecológica do IBGE (Recor), Brasília, DF. As setas indicam período de moderada a intensa herbivoria.

Figure 5. Percentage of canopy cover \pm standard deviation $(\bullet)$ and percentage of plants producing buds $(\triangle)$ of brevidecíduous (BDC) woody species of a "cerrado" stricto sensu of Reserva Ecológica do IBGE (Recor), Brasília, DF. Arrows indicate moderated to strong herbivory. 


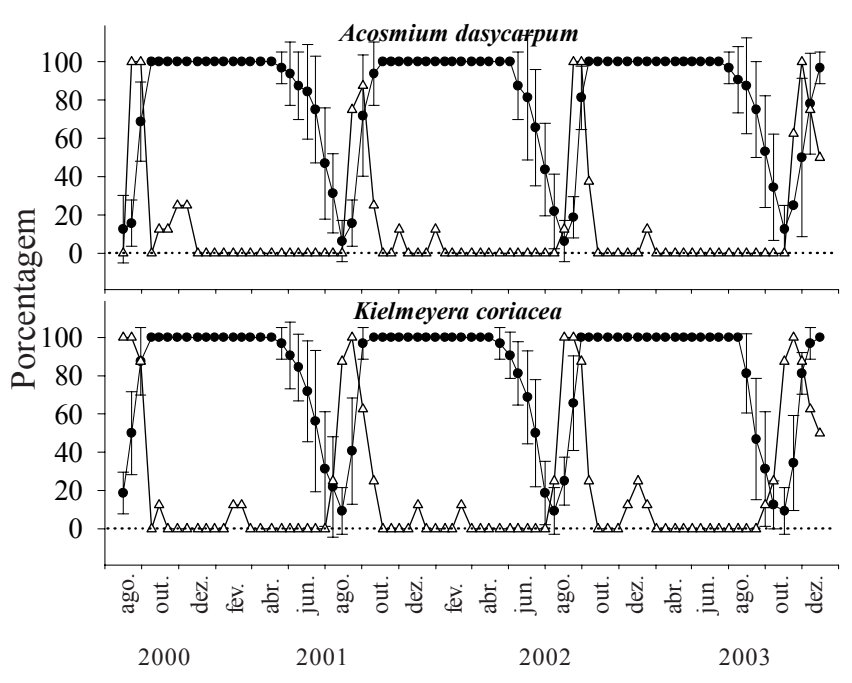

Figura 6. Porcentagem de cobertura de copa \pm desvio padrão $(\bullet)$ e de indivíduos brotando $(\triangle)$ de espécies lenhosas com comportamento fenológico decíduo (DEC) de um cerrado sentido restrito na Reserva Ecológica do IBGE (Recor), Brasília, DF.

Figure 6. Percentage of canopy cover \pm standard deviation $(\bullet)$ and percentage of plants producing buds $(\triangle)$ of deciduous (DEC) woody species of a "cerrado" stricto sensu of Reserva Ecológica do IBGE (Recor), Brasília, DF.

na transição entre os períodos seco e chuvoso. Esta evidente sazonalidade fenológica vegetativa foi também observada em outras comunidades e populações de espécies lenhosas de cerrado no Distrito Federal (Morais et al. 1995, Oliveira 1998, Oliveira \& Gibbs 2000), em São Paulo (Mantovani \& Martins 1988), bem como para áreas adjuntas de cerrado no Pará (Miranda 1995). Portanto, com base na dinâmica foliar do componente lenhoso, nós sugerimos a classificação desta vegetação de cerrado, bem como as demais comunidades de cerrado estudadas, como semidecidual sazonal.

Somente Miconia albicans, M. fallax e Myrsine guianensis apresentaram comportamento vegetativo distinto do padrão observado nas demais espécies estudadas. Nestas três espécies a produção de folhas foi reduzida ou ausente durante o período seco e se iniciou somente após o início das chuvas, prolongando-se durante todo o período chuvoso. Miconia albicans possui raízes superficiais (Oliveira 2004), bem como M. ferruginata, espécie cuja produção de folhas é também concentrada no período chuvoso (Franco et al. 2005). Estes resultados sugerem que $M$. albicans, M. fallax e M. guianensis podem ter a produção de folhas limitada pela disponibilidade de água no solo durante o período seco, provavelmente devido ao sistema radicular superficial. No entanto este fenômeno aguarda estudos específicos e mais detalhados para sua confirmação.

Durante o período seco no cerrado a demanda evaporativa atmosférica e a incidência de radiação solar aumentam (Miranda et al. 1997, Franco 1998, Meinzer et al. 1999) e afetam o controle estomático e a transpiração das plantas lenhosas. Quando estas condições se fizeram presentes durante o transcorrer do presente estudo, a maioria das espécies estudadas apresentou elevada proporção de folhas senescentes. Folhas neste estágio de desenvolvimento perdem a capacidade de controlar a abertura estomática, causando à redução da capacidade fotossintética e da eficiência no uso da água (Reich \& Borchert 1988, Borchert et al. 2002). Em ambientes tropicais sazonais a abscisão de folhas velhas na estação climática menos favorável, reduz a perda de água e maximiza-se a eficiência de aquisição de nutrientes pela planta (Reich \& Borchert 1984, Reich \& Borchert 1988).

Dessa forma, apesar das espécies lenhosas de cerrado possuírem a capacidade de controlar a abertura estomática em resposta à limitação hídrica atmosférica (Naves-Barbiero et al. 2000, Franco 2002, Bucci et al. 2004), a perda ou substituição da folhagem no período seco pode atuar como um mecanismo adicional na redução da perda de água nas espécies SVS, BDC e DEC. Além do mais, algumas espécies decíduas e semidecíduas de climas tropicais sazonais são capazes de produzir nova coorte de folhas dentro do período seco, graças à perda da folhagem mais velha, levando à redução de superfície de transpiração e, conseqüentemente, à restituição de níveis internos de água na planta (Reich \& Borchert 1984, Borchert 1994). Este mecanismo interno do controle hídrico foi sugerido por Franco (2002) para espécies lenhosas de cerrado e parece estar ocorrendo para as espécies SVS, BDC e DEC, acompanhadas em nosso estudo.

Folhas jovens são também mais suscetíveis à lixiviação de nutrientes pela água das chuvas (Sarmiento et al. 1985). Desse modo, a intensa produção de folhas no final do período seco ou no início do chuvoso, quando os índices pluviométricos ainda são relativamente mais baixos, reduziria a perda de nutrientes via lixiviação (Sarmiento et al. 1985).

O padrão de floração encontrado, ou seja, espécies florescendo ao longo de todo ano, porém marcado por um período de intensa floração na transição entre o período seco e chuvoso, também foi descrito para outras comunidades lenhosas de cerrado (Mantovani \& Martins 1998, Oliveira 1998, Batalha \& Mantovani 2000, Oliveira \& Gibbs 2000). Em nível específico, a floração anual e 


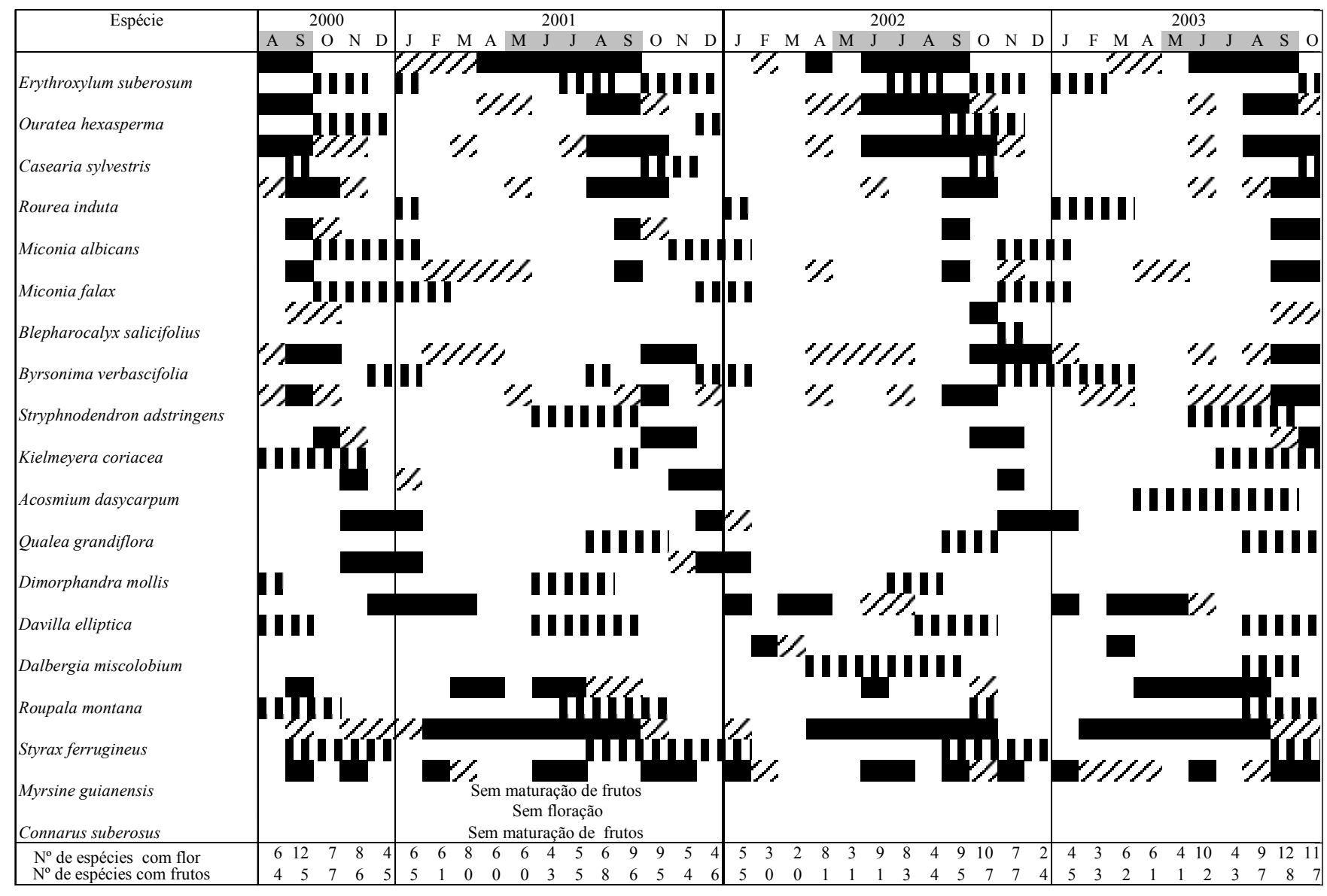

Figura 7. Fenograma de floração e de maturação de frutos de espécies lenhosas de um cerrado sentido restrito na Reserva Ecológica do IBGE (Recor), Brasília, DF. Período seco ( $\square$ ); período com dois ou mais indivíduos com flores na população ( $\square$ ); período com apenas um indivíduo florescendo na população (ש) ; período de maturação dos frutos (业).

Figure 7. Phenology diagram depicting flowering and fruit maturation period of woody species of a "cerrado" stricto sensu of Reserva Ecológica do IBGE (Recor), Brasília, DF. Dry season ( $\square$ ); period of the year when two or more plants had flowers ( $\square$ ); period of the year when just one plant had flowers (ש); period of ripe fruits (III).

regular observada em 17 espécies aqui estudadas $(89 \%$ do total) é um outro fenômeno comumente descrito para espécies lenhosas de cerrado (Barros 1992, 1998, 2002, Proença \& Gibbs 1994, Felfili et al. 1999). As 19 espécies estudadas são entomófilas (Oliveira \& Gibbs 2000) e apresentaram geralmente floração em picos rápidos e sincrônicos entre indivíduos, exceto Styrax ferrugineus, que apresentou um período prolongado de floração. Estes resultados estão de acordo como as afirmações de Augspurger (1981) e Primack (1980) de que plantas polinizadas por insetos florescem sincronicamente aumentando a atração dos agentes polinizadores e promovendo o fluxo de pólen entre indivíduos.

Espécies autocóricas e anemocóricas de comunidades lenhosas de cerrado geralmente dispersam seus diásporos durante e ao final do período seco, respectivamente, enquanto a dispersão de espécies zoocóricas ocorre predominantemente durante o período chuvoso (Mantovani \& Martins 1988, Oliveira \& Moreira 1992, Miranda 1995, Batalha \& Mantovani 2000). Este padrão foi confirmado entre as espécies aqui estudadas. A dispersão de frutos anemocóricos é favorecida durante períodos mais secos em regiões com clima sazonal, uma vez que a baixa umidade do ar, o aumento nas velocidades dos ventos e a redução na cobertura de folhas na vegetação facilitam a abertura dos frutos e a dispersão das sementes (Frankie et al. 1974, Oliveira \& Moreira 1992). Para Batalha \& Mantovani (2000) o dessecamento do pericarpo e a liberação de sementes em espécies autocóricas de cerrado também depende da baixa umidade durante o período seco. Já a maturação 
dos frutos zoocóricos ao longo do período chuvoso garante que estes se mantenham atrativos por períodos mais prolongados melhorando assim as chances de dispersão (Mantovani \& Martins 1988, Batalha \& Mantovani 2000).

Considerando os resultados do presente estudo e daqueles encontrados por outros autores (Mantovani \& Martins 1988, Batalha \& Mantovani 2000, Oliveira \& Gibbs 2000), pode-se sugerir que a restrição hídrica sazonal no cerrado não impede a ocorrência dos eventos fenológicos para a grande maioria das espécies lenhosas. Assim, as espécies lenhosas do cerrado são capazes de manter a produção de folhas e flores e frutos durante $o$ período seco, corroborando os resultados de diferentes estudos, que indicam haver água disponível para as plantas mesmo durante a seca sazonal (Franco 1998, Jackson et al. 1999, Silva 2003, Oliveira et al. 2005).

Grupos Fenológicos - As espécies estudadas apresentaram distintos comportamentos fenológicos com diferentes graus de deciduidade. Há desde espécies estritamente sempre verdes (e.g. Miconia albicans), cuja produção de folhas se dá por períodos prolongados até espécies totalmente decíduas (e.g. Kielmeyera coriacea), em que os períodos de produção e perda de folhas são bem marcados. No entanto, em certos casos o estabelecimento de grupos fenológicos vegetativos bem definidos é dificultado por variações que ocorrem entre populações. Por exemplo, Roupala montana e Qualea grandiflora foram classificadas por Franco et al. (2005) como SVC e DE, respectivamente, enquanto no presente estudo estas duas espécies foram categorizadas como SVS e BDC, respectivamente. Em um outro estudo realizado por Morais et al. (1995), Erythroxylum suberosum foi classificada como SVS, se considerarmos o mesmo critério empregado em nosso estudo, no entanto esta espécie foi aqui classificada como BDC.

Variações no comportamento fenológico entre indivíduos de uma mesma população, detectadas em nosso estudo, também dificultaram a caracterização do comportamento fenológico de algumas espécies. Segundo Borchert (1998), mudanças no comportamento fenológico de espécies lenhosas tropicais podem ocorrer em resposta a distintas condições climáticas e edáficas locais. Conforme proposto por Sarmiento \& Monasterio (1983) e por Oliveira (1998), os eventos fenológicos de espécies de savanas neotropicais são estratégias adaptativas que maximizariam a reprodução em ambientes tipicamente sazonais. Dessa forma, as variações fenológicas observadas entre populações e indivíduos podem representar a plasticidade fenotípica das espécies frente às mudanças locais na disponibilidade de recursos.

Os resultados deste e de outros estudos já mostram que espécies com substituição da folhagem durante o período seco, independente do grupo fenológico ao qual pertençam, são maioria dentro do componente lenhoso do cerrado (Miranda 1995, Morais et al. 1995, Oliveira 1998, Franco et al. 2005). Segundo Franco et al. (2005), folhas novas produzidas na estação seca possuem maiores concentrações de $\mathrm{N}$ e $\mathrm{P}$ causando aumento na eficiência de carboxilação das folhas e conseqüentemente a maior eficiência no uso de água pelas plantas. Dessa forma, a troca parcial ou total da folhagem em períodos hídricos menos favoráveis parece ser uma estratégia adaptativa que aumenta a eficiência no uso de água e que por isso ocorre com maior freqüência entre as espécies lenhosas de cerrado.

Agradecimentos - Ao CNPq pelo suporte financeiro por meio de bolsa de doutorado para o primeiro autor. Aos Programas: PELD/CNPq, SITIO 3 Recor-IBGE; IAI CRN 040; LBA CD05 \& LC16 pelo suporte financeiro. Ao Departamento de Ecologia da UnB e a toda direção e funcionários da Reserva Ecológica do IBGE (Recor) pelo apoio logístico. A José Roberto Rodrigues Pinto pela valiosa revisão da versão preliminar do artigo.

\section{Referências bibliográficas}

ANDRADE, L.A.Z., FELFILI, J.M. \& VIOLATTI, L. 2002. Fitossociologia de uma área de cerrado denso na RecorIBGE, Brasília, DF. Acta Botanica Brasilica 16:225-240.

AUGSPURGER, C.K. 1981. Reproductive synchrony of a tropical plant: experimental effects of pollinators and seed predators on Hybanthus prunifolius (Violaceae). Ecology 62:775-788.

BATALHA, M.A. \& MANTOVANI, W. 2000. Reproductive phenology patterns of cerrado plant species at the Pé-de-Gigante Reserve (Santa Rita do Passa Quatro, SP, Brazil): a comparison between the herbaceous and woody flora. Revista Brasileira de Biologia 60:129-145.

BARROS, M.A.G. 1992. Fenologia da floração, estratégias reprodutivas e polinização de espécies simpátricas do gênero Byrsonima Rich. (Malpighiaceae) Revista Brasileira de Biologia 52:343-353.

BARROS, M.A.G. 1998. Sistemas reprodutivos e polinização em espécies simpátricas de Erythroxylum (Erythroxylaceae) do Brasil. Revista Brasileira de Botânica 21:159-166.

BARROS, M.A.G. 2002. Floração sincrônica e sistemas reprodutivos em quatro espécies de Kielmeyera Mart. (Guttiferae). Acta Botanica Brasilica 16:113-122. 
BORCHERT, R. 1994. Soil and stem water storage determine phenology and distribution of tropical dry forest trees. Ecology 75:1437-1449.

BORCHERT, R. 1998. Responses of tropical trees to rainfall seasonality and its long-term changes. Climate Change 39:381-393.

BORCHERT, R., RIVERA, G. \& HAGNAUER, W. 2002. Modification of vegetative phenology in a tropical semideciduous forest by abnormal drought and rain. Biotropica 34:27-39.

BUCCI, S.J., GOLDSTEIN, G., MEINZER, F.C., FRANCO, A.C., CAMPANELLO, P. \& SCHOLZ, F.G. 2004. Mechanisms contributing to seasonal homeostasis of minimum leaf water potential and predawn disequilibrium between soil and plant water potential in Neotropical savanna trees. Trees 19:296-304.

EITEN, G. 1972. The cerrado vegetation of Brazil. The Botanical Review 38:201-341.

FELFILI, J.M., SILVA JUNIOR, M.C., DIAS, B.J. \& REZENDE, A.V. 1999. Estudo fenológico de Stryphnodendron adstringens (Mart.) Coville no cerrado sensu stricto da Fazenda Água Limpa no Distrito Federal, Brazil. Revista Brasileira de Botânica 22:83-90.

FOURNIER, L.A. 1974. Un método cuantitativo para la medición de características fenológicas em árboles. Turrialba 24:422-423.

FRANCO, A.C. 1998. Seasonal patterns of gas exchange, water relations and growth of Roupala montana, an evergreen savanna species. Plant Ecology 136:69-76.

FRANCO, A.C. 2002. Ecophysiology of woody plants. In The cerrados of Brazil (P.S. Oliveira \& R.J. Marquis, eds.). Columbia University Press, New York, p.178-197.

FRANCO, A.C., BUSTAMANTE, M., CALDAS, L.S., GOLDSTEIN, G., MEINZER, F.C., KOZOVITS, A.R., RUNDEL, P. \& CORADIN, V.R.T. 2005. Leaf functional traits of Neotropical savanna trees in relation to seasonal water deficit. Trees 19:326-335.

FRANKIE, G.W., BAKER, H.G. \& OPLER, P.A. 1974. Comparative phenological studies of trees in the lowlands of Costa Rica. Journal of Ecology 62:881-919.

FURLEY, P.A. \& RATTER, J.A. 1988. Soil resources and plant communities of the central Brazilian cerrado and their development. Journal of Biogeography 15:97-108.

HARIDASAN, M. 1993. Solos do Distrito Federal. In Cerrado: caracterização ocupação e perspectivas. (M. NovaisPinto, org.). Editora UnB/Sematec, Brasília, p.309-330.

JACKSON, P.C., MEINZER, F.C., BUSTAMANTE, M., GOLDSTEIN, G., FRANCO, A.C., RUNDEL, P.W., CALDAS, L.S., IGLER, E. \& CAUSIN, F. 1999. Partitioning of soil water among tree species in a Brazilian Cerrado. Tree Physiology 19:717-724.

LENZA, E. 2005. Fenologia, demografia foliar e características foliares de espécies lenhosas em um cerrado sentido restrito no Distrito Federal e suas relações como as condições climáticas. Tese de doutorado, Universidade de Brasília, Brasília.
MANTOVANI, W. \& MARTINS, F.R. 1988. Variações fenológicas das espécies de cerrado da Reserva Biológica de Mogi Guaçu. Estado de São Paulo. Revista Brasileira de Botânica 11:101-112.

MEINZER, F.C., GOLDSTEIN, G., FRANCO, A.C., BUSTAMANTE, M., IGLER, E., JACKSON, P. \& RUNDEL, P.W. 1999. Atmospheric and hydraulic limitations on transpiration in Brazilian cerradão woody species. Functional Ecology 13:273-282.

MIRANDA, I.S. 1995. Fenologia do estrato arbóreo de uma comunidade de cerrado em Alter-do-Chão. Revista Brasileira de Botânica 18:235-240.

MIRANDA, H.S., BUSTAMANTE, M. \& MIRANDA, A.C. 2002. The fire factor. In The cerrados of Brazil (P.S. Oliveira \& R.J. Marquis, eds.). Columbia University Press, New York, p.51-68.

MIRANDA, A.C., MIRANDA, H.S., LLOYD, J., GRACE, J., FRANCEY, R.J., MCINTYRE, J.A., MEIR, P., RIGGAN, P., LOCKWOOD, R. \& BRASS, J. 1997. Fluxes of carbon water and energy over Brazilian cerrado: an analysis using eddy covariance and stable isotopes. Plant Cell and Environment 20:315-328.

MORAIS, H.C., DINIZ, I.R. \& BAUMGARTEN, L. 1995. Padrões de produção de folhas e sua utilização por larvas de Lepidoptera em um cerrado de Brasília. Revista Brasileira de Botânica 18:163-170.

MOREIRA, A.G. 2000. Effects of fire protection on savanna structure in central Brazil. Jounal of Biogeography 27:1021-1029.

NAVES-BARBIERO, C.C., FRANCO, A.C., BUCCI, S.J. \& GOLDSTEIN, G. 2000. Fluxo de seiva e condutância estomática de duas espécies lenhosas sempre-verdes no campo sujo e cerradão. Revista Brasileira de Fisiologia Vegetal 12:119-134.

NEWSTROM, L.E. \& FRANKIE, G.W. 1994. A new classification for plant phenology based on flowering patterns in lowland tropical rain forest trees at La Selva, Costa Rica. Biotropica 26:141-159.

NUNES, R.V., SILVA JÚNIOR, M.C., FELFILI, M.J. \& WALTER, B.M.T. 2002. Intervalos de classe para abundância, dominância e freqüência do componente lenhoso do cerrado sentido restrito no Distrito Federal. Revista Árvore 26:173-182.

OLIVEIRA, P.E. 1998. Fenologia e biologia reprodutiva das espécies de cerrado. In Cerrado: ambiente e flora (S.M. Sano \& S.P. Almeida, eds.). Embrapa-CPAC, Planaltina, p.169-192.

OLIVEIRA, P.E. \& GIBBS, P.E. 2000. Reproductive biology of woody plants in a cerrado community of the central Brazil. Flora 195:311-329.

OLIVEIRA, P.E. \& MOREIRA, A.G. 1992. Anemocoria em espécies de cerrado e de mata de galeria. Revista Brasileira de Botânica 15:163-174.

OLIVEIRA, R.S. 2004. Comparative water use and water acquisition strategies of trees from the Brazilian Cerrado and Amazônia. Ph.D thesis, University of California, Berkeley. 
OLIVEIRA, R.S., BEZERRA, L., DAVIDSON, E.A., PINTO, F., KLINK, C.A., NEPSTAD, D.C. \& MOREIRA, A. 2005. Deep root function in soil water dynamics in cerrado savannas of central Brazil. Functional Ecology 19:574-581.

OLIVEIRA FILHO, A.T. \& RATTER, J.A. 2002. Vegetation physionomies and woody flora of the cerrado biome. In The cerrados of Brazil (P.S. Oliveira \& R.J. Marquis, eds.). Columbia University Press, New York, p.91-120.

PRIMACK, R.B. 1980. Phenological variation within natural populations: flowering in New Zealand montane shrubs. Journal of Ecology 68:849-862.

PROENÇA, C.E.B. \& GIBBS, P.E. 1994. Reproductive biology of eight sympatric Myrtaceae from central Brazil. New Phytologist 126:343-354.

RATTER, J.A., BRIDGEWATER, S. \& RIBEIRO, J.F. 2003. Analysis of the floristic composition of the Brazilian cerrado vegetation III: comparisons of woody vegetation of 376 areas. Edinburgh Journal of Botany 60:57-109.

REICH, P.B. \& BORCHERT, R. 1984. Water stress and tree phenology in a tropical dry forest in the lowlands of Costa Rica. Journal of Ecology 72:61-74.
REICH, P.B. \& BORCHERT, R. 1988. Changes with leaf age in stomatal function and water status of several tropical tree species. Biotropica 20:60-69.

RIBEIRO, J.F. \& WALTER, B.M.T. 1998. Fitofisionomias do bioma cerrado. In Cerrado: ambiente e flora (S.M. Sano \& S.P. Almeida, eds.). Embrapa-CPAC, Planaltina, p.89-166.

SARMIENTO, G. 1984. The ecology of neotropical savannas. Harvard University Press, Cambridge.

SARMIENTO, G. \& MONASTERIO, M. 1983. Life forms and phenology. In Ecosystems of the world: tropical savannas (F. Bouliere, ed.). Elsevier, Amsterdan, p.79-108.

SARMIENTO, G., GOLDSTEIN, G. \& MEINZER, F. 1985. Adaptative strategies of woody species in neotropical savannas. Biological Review 60:315-355.

SILVA, L.B.P. 2003. Disponibilidade de água para as plantas e evapotranspiração em um cerrado denso, um cerrado strictu sensu e uma pastagem plantada. Dissertação de mestrado, Universidade de Brasília, Brasília.

VAN DER PIJL, L. 1982. Principles of dispersal in higher plants. Springer Verlag, New York. 